\title{
Four-nucleon scattering: a new numerical approach
}

\author{
A. Deltuva ${ }^{a *}$ and A.C. Fonseca ${ }^{\mathrm{a}}$ \\ ${ }^{a}$ Centro de Física Nuclear, University of Lisbon, \\ Av. Prof. Gama Pinto 2, 1649-003 Lisbon, Portugal
}

The AGS equations are solved for $n^{3} \mathrm{H}$ and $p^{3} \mathrm{He}$ scattering including the Coulomb interaction. Comparison with previous work confirms the accuracy of the calculation and helps clarify a number of issues related to the $n^{3} \mathrm{H}$ total cross section at the peak of the resonance region, as well as an $A_{y}$ deficiency in $p^{3} \mathrm{He}$. Calculations are fully converged in terms of $N N$ partial waves and involve no uncontrolled approximations.

\section{INTRODUCTION}

In spite of the progress that has taken place over the years on the solution of the threenucleon scattering problem in terms of ab initio calculations, there are still some open problems that defy our understanding. They are the $A_{y}$ puzzle in elastic $N d$ scattering and the space star anomaly in $N d$ breakup, just to name a few. These problems persist even when the Coulomb interaction is added [1,2,3] and seem to be insensitive to the choice of realistic $2 N+3 N$ force model. As it has already been highlighted in the past [4, 5,6], the four-nucleon scattering problem reveals further discrepancies between theory and experiment that need clarification in terms of improved calculations using modern force models and efficient numerical algorithms that allow for a numerically converged solution of the Alt, Grassberger and Sandhas (AGS) equation [7] for the transition operators.

In four-nucleon scattering the Coulomb interaction is paramount to treat $p^{3} \mathrm{He}$, to separate the $n^{3} \mathrm{He}$ threshold from $p^{3} \mathrm{H}$ and at the same time avoid a second excited state of the alpha particle a few keV bellow the lowest scattering threshold [8]. Given the success recently achieved in including the Coulomb interaction in $p d$ elastic scattering and breakup [2,3], we generalize the proposed method to the four-nucleon system and study $p^{3}$ He below three-body breakup threshold.

The work we present here constitutes the first effort to design and construct a new scattering code that may bring the treatment of the four-nucleon system to the same degree of accuracy and sophistication as we already have in the three-nucleon system. The symmetrized AGS equations for four identical nucleons are given by

$\mathcal{U}^{11}=-\left(G_{0} t G_{0}\right)^{-1} P_{34}-P_{34} U G_{0} t G_{0} \mathcal{U}^{11}+\tilde{U} G_{0} t G_{0} \mathcal{U}^{21}$,

$\mathcal{U}^{21}=\left(G_{0} t G_{0}\right)^{-1}\left(1-P_{34}\right)+\left(1-P_{34}\right) U G_{0} t G_{0} \mathcal{U}^{11}$,

where $\mathcal{U}^{11}\left(\mathcal{U}^{21}\right)$ is the transition operator for $1+3 \rightarrow 1+3(1+3 \rightarrow 2+2), t$ the $N N$ t-matrix and $G_{0}$ the four free particle Green's function. The operators $U$ and $\tilde{U}$ are respectively

\footnotetext{
*Supported by the FCT grant SFRH/BPD/14801/2003
} 
$1+(3)$ and $(2)+(2)$ subsystem transition operators given by

$U=P G_{0}^{-1}+P t G_{0} U$,

$\tilde{U}=\tilde{P} G_{0}^{-1}+\tilde{P} t G_{0} \tilde{U}$,

where the permutation operators $P$ and $\tilde{P}$ are given by $P=P_{12} P_{23}+P_{13} P_{23}$, and $\tilde{P}=$ $P_{13} P_{24}$. Defining $\left|\phi_{1}\right\rangle$ and $\left|\phi_{2}\right\rangle$ the initial/final $(1+3)$ and $(2+2)$ states that satisfy the equations

$\left|\phi_{1}\right\rangle=G_{0} t P\left|\phi_{1}\right\rangle$,

$\left|\phi_{2}\right\rangle=G_{0} t \tilde{P}\left|\phi_{2}\right\rangle$,

the matrix elements $\left\langle\phi_{\alpha}\left|T^{\alpha \beta}\right| \phi_{\beta}\right\rangle=S_{\alpha}\left\langle\phi_{\alpha}\left|\mathcal{U}^{\alpha \beta}\right| \phi_{\beta}\right\rangle$ with $S_{1}=3$ and $S_{2}=2$ lead to the transition amplitudes from which one calculates observables. After partial wave decomposition we solve a three-variable integral equation with no approximations beyond the usual discretization of continuous variables in a finite mesh.

\section{RESULTS}

In order to calibrate the accuracy of our new numerical algorithm we calculate first the negative eigenvalues of the kernel that correspond to the ${ }^{4} \mathrm{He}$ binding energy. The Coulomb interaction is included through screening and charge dependence in the $N N$ interaction taken in isospin $T=0$ alone. In Table 1 we show the convergence of the results for the binding energy in terms of two-nucleon total angular momentum $I$. The calculation with $I \leq 6$ includes 1057 channels and clearly shows the accuracy of the numerical method we use. Results of ref. [12] for CD Bonn include total isospin $T=1$ and $T=2$ states, whereas they are neglected in our calculations.

Table 1

$\alpha$-particle binding energy as function of the total two-nucleon angular momentum $I$. Predictions for AV8' do not include Coulomb.

\begin{tabular}{l|cccccc|c} 
& $I \leq 1$ & $I \leq 2$ & $I \leq 3$ & $I \leq 4$ & $I \leq 5$ & $I \leq 6$ & refs. [9]10,11]12] \\
\hline AV8 & 23.08 & 25.16 & 25.69 & 25.85 & 25.90 & 25.91 & $25.90-25.94$ \\
AV18 & 22.30 & 23.75 & 24.15 & 24.20 & 24.23 & 24.24 & $24.22-24.25$ \\
CD Bonn & 25.03 & 25.95 & 26.07 & 26.10 & 26.11 & 26.11 & $26.13-26.16$ \\
INOY04 & 28.68 & 29.09 & 29.10 & 29.11 & 29.11 & 29.11 & 29.11
\end{tabular}

Next we show results for $n^{3} \mathrm{H}$ scattering by solving Eqs. (1a) and (1b) in isospin $T=1$. The phase shifts at $E_{n}=3.5 \mathrm{MeV}$ are shown in Table 2 together with the total cross section $\sigma_{t}$. The present work confirms previous calculations by the Grenoble and Pisa groups (second and third lines respectively) compiled in ref. [6] together with the results by one of us (fourth line) where the AV18 interaction was represented in rank one. The small differences between the phases in the first three lines are probably due to higher $N N$ partial waves $(I \leq 4)$ that are included in the present calculation together with $\ell_{y}, \ell_{z} \leq 4$. 
Table 2

$n^{3} \mathrm{H}$ phase shifts and mixing parameter at $E_{n}=3.5 \mathrm{MeV}$ together with total cross section.

\begin{tabular}{|c|c|c|c|c|c|c|c|c|c|}
\hline & $0^{+}$ & $0^{-}$ & $1^{+}$ & \multicolumn{3}{|c|}{$1^{-}$} & $2^{-}$ & \multicolumn{2}{|l|}{$\sigma_{t}(b)$} \\
\hline & $\left({ }^{1} \mathrm{~S}_{0}\right)$ & $\left({ }^{3} \mathrm{P}_{0}\right)$ & $\left({ }^{3} \mathrm{~S}_{1}\right)$ & $\left({ }^{3} \mathrm{P}_{1}\right)$ & $\left({ }^{1} \mathrm{P}_{1}\right)$ & $\delta$ & $\left({ }^{3} \mathrm{P}_{2}\right)$ & & \\
\hline AV 18 & -66.07 & 20.72 & -58.44 & 40.08 & 20.71 & -44.50 & 42.48 & 2.329 & this work \\
\hline & -66.5 & 20.9 & -58.5 & 37.3 & 20.7 & -43.5 & 41.0 & 2.24 & ref. [6] \\
\hline & -66.3 & 20.6 & -58.7 & 38.6 & 20.5 & -45.5 & 40.1 & 2.24 & ref. [6] \\
\hline & -63.7 & 27.5 & -58.3 & 44.8 & 24.7 & & 44.2 & 2.51 & ref. [6] \\
\hline CD-Bonn & -64.63 & 18.97 & -57.41 & 39.44 & 20.19 & -44.95 & 42.45 & 2.282 & this work \\
\hline
\end{tabular}

In spite of the improved accuracy, both AV18 and CD Bonn interactions lead to cross sections in the resonance region that fail to reproduce the experimental data. This is shown in Fig. 1,

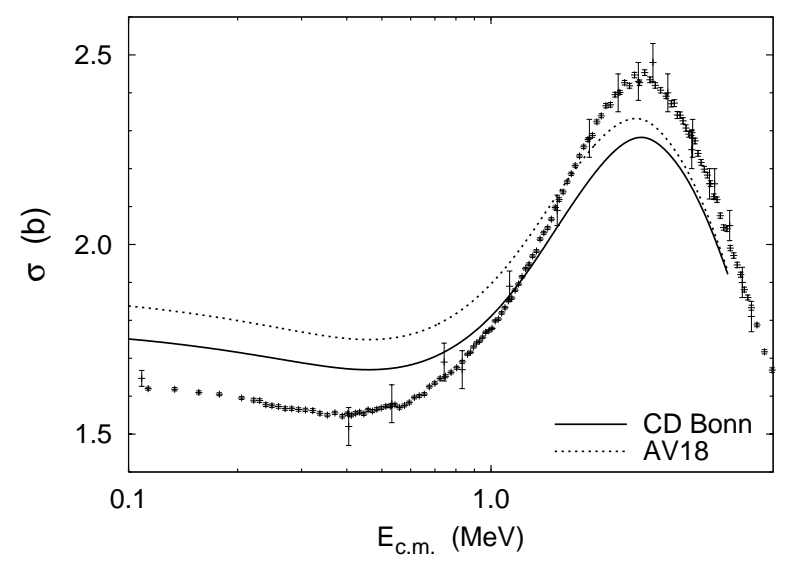

Figure 1. Total cross section for $n^{3} \mathrm{H}$ scattering versus center of mass energy for different realistic interactions.

Finally we use the method developed for $p d$ elastic scattering [2,3] to include the Coulomb interaction between the protons. This means using the two-potential formula

$\left\langle\phi_{1} \vec{p}_{f}\left|T^{11}\right| \phi_{1} \vec{p}_{i}\right\rangle=\left\langle\vec{p}_{f}\left|t_{C}\right| \vec{p}_{i}\right\rangle+\lim _{R \rightarrow \infty}\left\{Z_{R}^{-\frac{1}{2}}\left(p_{f}\right)\left\langle\phi_{1} \vec{p}_{f}\left|\left[T_{(R)}^{11}-t_{R}\right]\right| \phi_{1} \vec{p}_{i}\right\rangle Z_{R}^{-\frac{1}{2}}\left(p_{i}\right)\right\}$,

where the first term is the long range Coulomb amplitude between the proton and the center of mass of ${ }^{3} \mathrm{He}$ and the second term represents the Coulomb modified nuclear short range amplitude that results from the difference between the matrix elements of the AGS operator $T_{(R)}^{11}$ calculated with screened Coulomb between the three protons and the two body $p^{3}$ He screened Coulomb t-matrix $t_{R}$ after renormalization with $Z_{R}^{-\frac{1}{2}}$. 

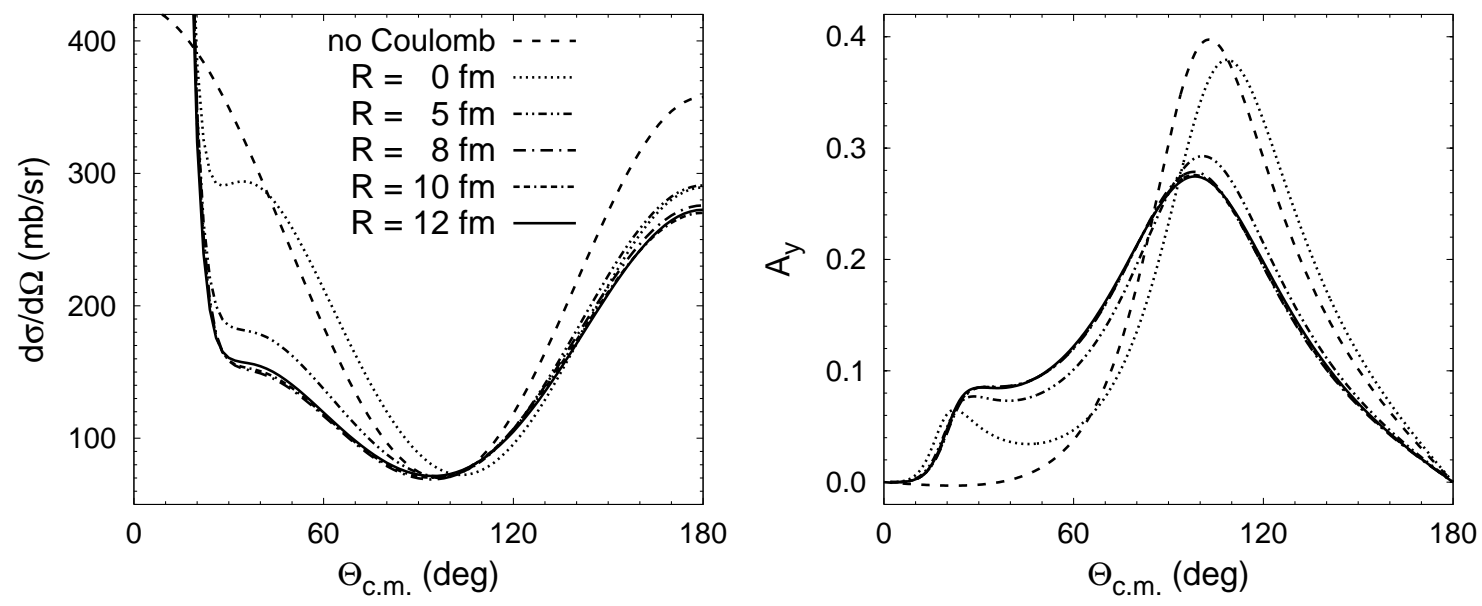

Figure 2. Convergence of $p^{3} \mathrm{He}$ observables at $E_{p}=4 \mathrm{MeV}$ with the screening radius $R$ of the Coulomb potential.
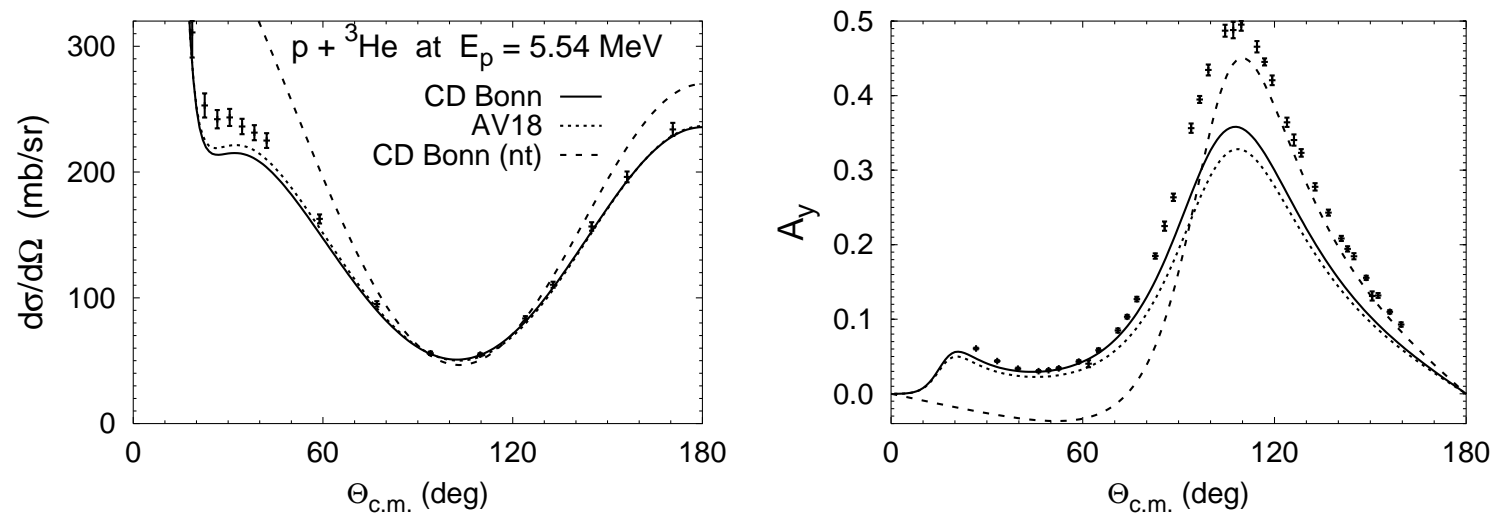

Figure 3. Differential cross section and nucleon analyzing power for $p^{3} \mathrm{He}$ scattering at $E_{p}=5.54 \mathrm{MeV}$ using CD Bonn (solid) and AV18 (dotted) potentials plus the Coulomb interaction between all three protons. The dashed line corresponds to CD Bonn alone. Experimental data are from refs. [13, 14].

The convergence of the calculation with the screening radius $R$ is shown in Fig. 2. For $R=12 \mathrm{fm}$ we get converged results for both observables. Further results are shown in Figs. 3 and 4 for the $d \sigma / d \Omega, A_{y}, C_{x x}$ and $C_{y y}$ at $E_{p}=5.54 \mathrm{MeV}$. The calculations show that there are large Coulomb effects, and greater sensitivity to the $N N$ interaction compared to what is observed in $p d$ scattering at low energy. The calculated analyzing power $A_{y}$ shows a large discrepancy with the data but $C_{x x}$ and $C_{y y}$ are in reasonable agreement with experiment. 

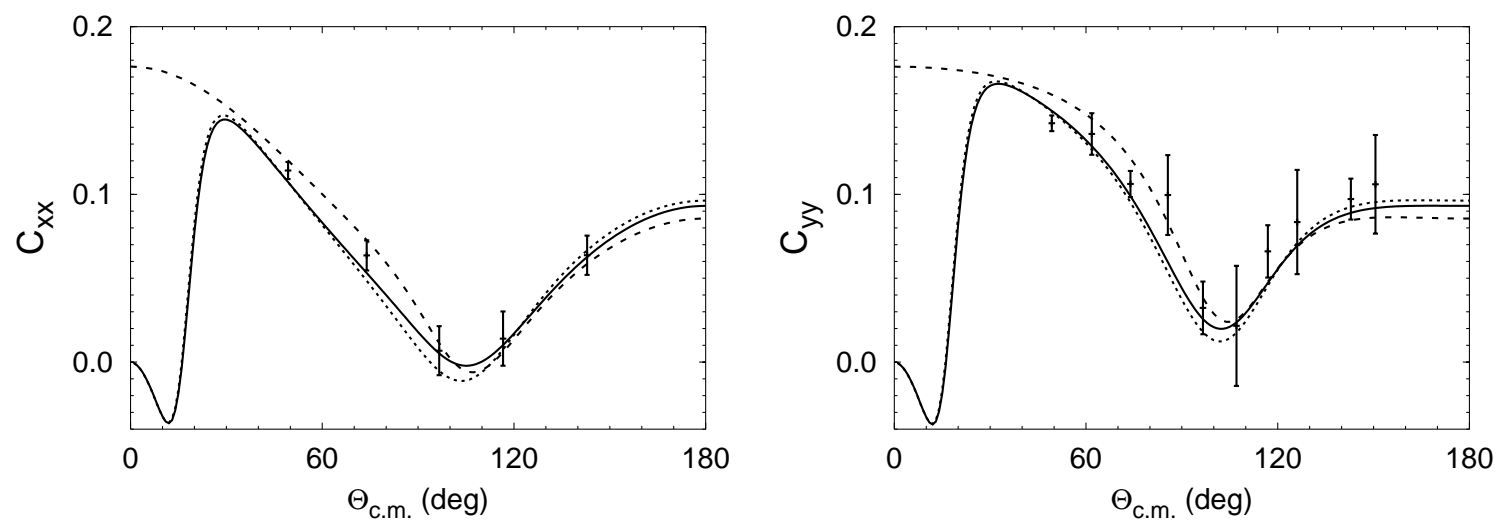

Figure 4. Spin correlation coefficients for $p^{3} \mathrm{He}$ scattering at $E_{p}=5.54 \mathrm{MeV}$. Curves as in Fig. 3. Experimental data are from ref. [14].

\section{REFERENCES}

1. A. Kievsky, M. Viviani, S. Rosati, Phys. Rev. C64 (2001) 024002.

2. A. Deltuva, A. C. Fonseca, P. U. Sauer, Phys. Rev. C71 (2005) 054005.

3. A. Deltuva, A. C. Fonseca, P. U. Sauer, Phys. Rev. C72 (2005) 054004.

4. A. C. Fonseca, Phys. Rev. Lett. 83 (1999) 4021.

5. M. Viviani et al., Phys. Rev. Lett. 86 (2001) 3739.

6. R. Lazauskas et al., Phys. Rev. C71 (2005) 034004.

7. P. Grassberger and W. Sandhas, Nucl. Phys. B2 (1967) 181; E. O. Alt, P. Grassberger, W. Sandhas, JINR report n1E4-6688 (1972).

8. A. C. Fonseca, G. Hale, J. Haidenbauer, Few-Body Systems 31 (2002) 139.

9. A. Nogga et al., Phys. Rev. C65 (2001) 054003.

10. H. Kamada et al., Phys. Rev. C64 (2001) 044001.

11. R. Lazauskas and J. Carbonell, Phys. Rev. C70 (2004) 044002.

12. M. Viviani, L. E. Marcucci, S. Rosati, A. Kievsky, L. Girlanda, Few-Body Systems 39 (2006) 159.

13. D. G. McDonald, W. Haeberli, L. W. Morrow, Phys. Rev. 133 (1964) B1178.

14. M. T. Alley and L. D. Knutson, Phys. Rev. C48 (1993) 1890. 